OPEN ACCESS

Edited by:

Jonathan Paul Mynard, Royal Children's Hospital, Australia

Reviewed by:

Federico Gutierrez-Larraya, University Hospital La Paz, Spain Hoang H. Nguyen,

University of Texas Southwestern Medical Center, United States

${ }^{*}$ Correspondence: Cheng Wang

wangcheng2nd@csu.edu.cn orcid.org/0000-0002-7120-0654

Specialty section: This article was submitted to Pediatric Cardiology, a section of the journal

Frontiers in Pediatrics

Received: 30 October 2020 Accepted: 03 February 2021

Published: 03 March 2021

Citation: Zou R, Wang S, Li F, Lin P, Zhang J, Wang Y, Xu Y and Wang C (2021) The Application of Head-Up Tilt Test to Diagnose Hemodynamic Type of Orthostatic Intolerance in Children Aged Between 3 and 5 Years.

Front. Pediatr. 9:623880. doi: $10.3389 /$ fped.2021.623880

\section{The Application of Head-Up Tilt Test to Diagnose Hemodynamic Type of Orthostatic Intolerance in Children Aged Between 3 and 5 Years}

\author{
Runmei Zou ${ }^{1}$, Shuo Wang ${ }^{2}$, Fang $\mathrm{Li}^{1}$, Ping Lin ${ }^{1}$, Juan Zhang ${ }^{1}$, Yuwen Wang ${ }^{1}$, Yi Xu ${ }^{1}$ and \\ Cheng Wang ${ }^{1 *}$ \\ 1 Department of Pediatric Cardiovasology, Children's Medical Center, The Second Xiangya Hospital, Central South University, \\ Changsha, China, ${ }^{2}$ Jishou University School of Medicine, Jishou, China
}

Objectives: The head-up tilt test (HUTT) is a useful tool to assess autonomic function and to reproduce neurally mediated reflex. In this study, we evaluated the use of HUTT in pediatric patients aged 3-5 years with orthostatic intolerance.

Materials and Methods: The medical history and HUTT records of 345 (180 males, aged from 3 to 5 years) cases of patients who complained of symptoms of orthostatic intolerance and who visited the Syncope Ward, Children's Medical Center, The Second Xiangya Hospital, Central South University from January 2003 to December 2019, were reviewed retrospectively.

Results: Seventy-nine (22.9\%) cases had positive responses to complete HUTT (basic HUTT and sublingual nitroglycerin HUTT), while 29 (8.4\%) cases had positive responses if only basic HUTT was performed. Sublingual nitroglycerin provocation significantly increased the positive rate of the test $\left(x^{2}=27.565, P<0.001\right)$. The most frequent hemodynamic response to HUTT was vasoinhibitory type vasovagal syncope (12.2\%), Syncope (28.7\%), and dizziness (22.6\%) were the most common symptoms. Eight cases discontinued the test due to intolerable symptoms without severe adverse events occurring.

Conclusions: HUTT was safe and well-tolerated and could be used to diagnose the hemodynamic type of orthostatic intolerance in children aged 3-5 years.

Keywords: orthostatic intolerance, neurally mediated syncope, head-up tilt test, children, hemodynamic type

\section{INTRODUCTION}

Orthostatic intolerance is common in children and adolescents, and is defined as individuals who have difficulty in tolerating an upright posture and who present signs and symptoms of transient loss of consciousness, fatigue, headaches, lightheadedness, visual disturbances, sweating, vomiting, nausea, abdominal pains, and so on when standing (1). These symptoms and signs can be abated when returned to the supine position. The mechanism involves an inability to maintain adequate venous return to the heart due to venous pooling, primarily in the lower body, during standing. Orthostatic intolerance can be diagnosed as postural tachycardia syndrome (POTS), neurocardiogenic syncope, orthostatic hypotension, and vasovagal syncope (2). 
A comprehensive history, physical examination, standing test, and electrocardiogram (ECG) are necessary for diagnosis.

The head-up tilt test (HUTT) is an important tool to assess autonomic function and is used for differential diagnosis of transient loss of consciousness and orthostatic intolerance in adults and children $(3,4)$. HUTT can be used to diagnose the hemodynamic type of orthostatic intolerance (5). HUTT data in pediatric patients under the age of 6 years are limited. In this study, we evaluate the clinical use of HUTT in children aged between 3 and 5 years with orthostatic intolerance.

\section{METHODS}

\section{Participants}

We retrospectively reviewed 345 (180 males, aged between 3 and 5 years) cases of patients who complained of orthostatic intolerant symptoms of syncope, headaches, dizziness, visual disturbances, sweating, vomiting, nausea, abdominal pains, and so on when standing upright, and who visited the Syncope Ward, Children's Medical Center, The Second Xiangya Hospital, from January 2003 to December 2019. The demographic and clinical data including HUTT records were reviewed. Heart, pulmonary, cerebral, and other system diseases were excluded after an initial evaluation consisting of history, physical examination, baseline laboratory testing, ECG, Holter ECG, echocardiography, chest Xray, electroencephalogram, and cranial CT or MRI. According to the age range, patients were divided into three groups: group 3-4 referred to those aged above or equal to 3 and $<4$ years, group $4-$ 5 referred to those aged above or equal to 4 and $<5$ years, group $5-6$ referred to those aged above or equal to 5 and $<6$ years.

\section{HUTT Protocol}

The HUTT consisted of two stages: basic HUTT and sublingual nitroglycerin HUTT. The protocol had been carried out according to a previous study $(6,7)$. HUTT was approved by the Ethics Committee of The Second Xiangya Hospital, Central South University. Informed consent was obtained directly from all the subjects or their guardians. The test was performed after overnight fasting between 8:00 a.m. and 12:00 p.m.. The subjects were asked to lay still for $10 \mathrm{~min}$, and then basic heart rate (HR), blood pressure (BP), and ECG were recorded. Subjects were tilted at $60^{\circ}$ head upward, and HR, BP, and ECG were recorded continuously until either $45 \mathrm{~min}$ duration, or the development of syncope or intolerable near syncope symptoms (basic HUTT). If syncope occurred, patients were rapidly placed in the supine position. If the subjects did not develop syncope or presyncope, they underwent nitroglycerin stimulated HUTT. A tilted posture was maintained, subjects were medicated with nitroglycerin, and HR, BP, and ECG were recorded for $20 \mathrm{~min}$ or until syncope or presyncope occurred.

Positive responses to HUTT included vasovagal syncope (VVS), postural tachycardia syndrome (POTS), orthostatic hypotension $(\mathrm{OH})$, and orthostatic hypertension $(\mathrm{OHT})$ (6). VVS was defined as the development of syncope or presyncope accompanied by hypotension (systolic $\mathrm{BP} \leq 80 \mathrm{mmHg}$ and/or diastolic BP $\leq 50 \mathrm{mmHg}$, or over $25 \%$ decrease in mean blood pressure), bradycardia ( $\mathrm{HR}<75 \mathrm{bpm}$ ), or cardiac arrest $>3 \mathrm{~s}$.
VVS was further classified into three responses: vasoinhibitory type VVS (significant reduction in BP but insignificant change in HR), cardioinhibitory type VVS (significant reduction in HR but insignificant change in BP), and mixed type VVS (significant reduction both in BP and HR). POTS was defined as dizziness, chest distress, headaches, palpitation, and pallor with an increase in HR $\geq 40 \mathrm{bpm}$ within $10 \mathrm{~min}$ of HUTT. OHT was defined as (within 3 min of HUTT) orthostatic intolerance symptoms and an increase in systolic BP $\geq 20 \mathrm{mmHg}$, and/or diastolic BP increments $\geq 25 \mathrm{mmHg}$ without an obvious change in HR. OH was defined as (within 3 min of HUTT) orthostatic intolerance symptoms and a decrease in systolic $\mathrm{BP} \geq 20 \mathrm{mmHg}$, and/or diastolic $\mathrm{BP} \geq 10 \mathrm{mmHg}$.

\section{Statistical Analysis}

Statistical analysis was performed by SPSS 17.0. Data were described as mean \pm SD for normally distributed continuous variables, median $\left(\mathrm{P}_{25}, \mathrm{P}_{75}\right)$ for non-normally distributed continuous variables, and percent prevalence for dichotomized variables. One-way analysis of variance (ANOVA) was used to analyze three or more normally distributed continuous variables. Manne-Whitney test was used to compare nonnormally distributed continuous variables among groups. $\chi^{2}$ test, or Fisher exact test was used to compare dichotomized variables. $P<0.05$ were considered statistically significant.

\section{RESULTS}

\section{Basic Characteristics of the Study Population}

There were 38 subjects [22 (57.9\%) males] aged between 3 and 4 years, 114 individuals [65 (57.0\%) males] aged between 4 and 5 years, and 193 subjects [93 (48.2\%) males] aged between 5 and 6 years. There were no significant differences in gender ratio, history duration, basic systolic, and diastolic blood pressure among the three groups, except for the fact that HR decreased, and body mass index increased with increasing age $(P=0.004$ and $P<0.001$, respectively) (Table 1).

\section{Presenting Symptoms in the Study Population}

The most common symptoms were syncope (28.7\%) and dizziness (22.6\%), followed by sighing (11.9\%), chest pain (8.4\%), palpitation (6.4\%), headaches (5.5\%), and fatigue (1.2\%). Other symptoms included visual disturbances, sweating, vomiting, nausea, and abdominal pains. The incidence of syncope decreased with increasing age $\left(x^{2}=8.929, P=0.012\right)$, whereas the incidence of dizziness increased with increasing age $\left(x^{2}=6.910\right.$, $P=0.032$ ) (Table 2).

\section{HUTT Results}

As shown in Table 3, 79 (22.9\%) cases had positive responses to complete HUTT (basic HUTT and sublingual nitroglycerin HUTT), while 29 (8.4\%) cases had positive responses if only basic HUTT was performed. Sublingual nitroglycerin provocation significantly increased the positive rate of the test ( 22.9 vs. $8.4 \%$, $\left.x^{2}=27.565, P<0.001\right)$. As shown in Table 4, hemodynamic types 
TABLE 1 | Basic characteristics of the study population.

\begin{tabular}{|c|c|c|c|c|c|c|c|}
\hline Age range & $\begin{array}{l}\text { No. } \\
(\%)\end{array}$ & $\begin{array}{c}\text { Male } \\
(\%)\end{array}$ & $\begin{array}{c}\text { BMI } \\
\left(\mathrm{kg} / \mathrm{m}^{2}\right)\end{array}$ & $\begin{array}{l}\text { Duration (mouth), } \\
\text { median (P25, P75) }\end{array}$ & $\begin{array}{c}\text { SBP } \\
(\mathrm{mmHg})\end{array}$ & $\begin{array}{c}\text { DBP } \\
(\mathrm{mmHg})\end{array}$ & $\begin{array}{c}\text { HR } \\
\text { (bpm) }\end{array}$ \\
\hline 3-4 Years & $38(11.0)$ & $22(57.9)$ & $12.7 \pm 5.7$ & $6.5(0.7,12.0)$ & $100.9 \pm 14.6$ & $62.6 \pm 10.0$ & $99.1 \pm 14.8$ \\
\hline 4-5 Years & $114(33.0)$ & $65(57.0)$ & $15.4 \pm 5.5$ & $2.0(0.7,6.3)$ & $98.8 \pm 13.1$ & $61.6 \pm 7.5$ & $95.4 \pm 13.4$ \\
\hline 5-6 Years & 193 (55.9) & $93(48.2)$ & $15.1 \pm 3.3$ & $2.0(0.5,12.0)$ & $99.9 \pm 9.8$ & $61.2 \pm 7.8$ & $88.3 \pm 14.0$ \\
\hline Total & 345 (100.0) & 180 (52.2) & $14.9 \pm 4.5$ & $2.0(0.63,12.0)$ & $99.7 \pm 11.5$ & $61.5 \pm 7.5$ & $91.9 \pm 14.4$ \\
\hline Statistic value & - & 2.800 & 5.526 & 0.960 & 0.523 & 0.626 & 11.317 \\
\hline$P$ & - & 0.247 & 0.004 & 0.619 & 0.593 & 0.535 & $<0.001$ \\
\hline
\end{tabular}

BMI, Body mass index; SBP, basic systolic blood pressure; DBP, diastolic blood pressure; HR, heart rate; bpm: beats per minute.

TABLE 2 | Presenting symptoms in the study population.

\begin{tabular}{|c|c|c|c|c|c|c|c|c|}
\hline Age range & Syncope & Dizziness & Sighing & Palpitation & Headache & Fatigue & Chest pain & Others \\
\hline 3-4 years & $18(47.4)$ & $4(10.5)$ & 5 (13.2) & $4(10.5)$ & 0 & 0 & $1(2.6)$ & $6(15.8)$ \\
\hline $4-5$ years & 35 (30.7) & $21(18.4)$ & 18 (15.8) & $9(7.9)$ & $7(6.14)$ & $2(1.8)$ & $7(6.1)$ & 15 (13.2) \\
\hline 5-6 years & 46 (23.8) & $53(27.5)$ & 18 (9.3) & $9(4.7)$ & $12(6.2)$ & $2(1.0)$ & 21 (10.9) & 32 (16.6) \\
\hline Total & 99 (28.7) & 78 (22.6) & $41(11.9)$ & $22(6.4)$ & $19(5.5)$ & $4(1.2)$ & $29(8.4)$ & 53 (15.4) \\
\hline$x^{2}$ value & 8.929 & 6.910 & 2.872 & 2.485 & 0.001 & 2.485 & 4.449 & 0.910 \\
\hline$P$ & 0.012 & 0.032 & 0.238 & 0.289 & 0.978 & 0.289 & 0.108 & 0.823 \\
\hline
\end{tabular}

Other symptoms referred to visual disturbances, sweating, vomiting, nausea, and abdominal pains.

TABLE 3 | Number of the patients with positive or negative responses to the complete HUTT or only basic HUTT.

\begin{tabular}{|c|c|c|c|c|c|c|}
\hline \multirow[t]{2}{*}{ Age range } & \multicolumn{2}{|c|}{ Positive } & \multicolumn{2}{|c|}{ Negative } & \multirow[t]{2}{*}{ Unfinished } & \multirow[t]{2}{*}{ Total } \\
\hline & Only basic HUTT & Complete HUTT & Only basic HUTT & Complete HUTT & & \\
\hline 3-4 years & 7 (18.4) & $11(28.9)$ & 30 (78.9) & $26(68.4)$ & $1(2.6)$ & 38 \\
\hline $4-5$ years & $9(7.9)$ & $23(20.2)$ & $99(86.8)$ & 85 (74.6) & $6(5.3)$ & 114 \\
\hline 5-6 years & $13(6.7)$ & 45 (23.3) & $179(92.7)$ & $147(76.2)$ & $1(0.5)$ & 193 \\
\hline Total & $29(8.4)$ & $79(22.9)^{\star}$ & 308 (89.3) & $258(74.8)$ & $8(2.3)$ & 345 \\
\hline
\end{tabular}

Complete HUTT referred that patients underwent two stages of HUTT (both basic HUTT and sublingual nitroglycerin HUTT).

${ }^{*}$ Compared with only basic HUTT, $P<0.001$.

TABLE 4 | Hemodynamic types in positive response to head-up tilt test.

\begin{tabular}{lccccc}
\hline Age range & No. & VVS-V & VVS-C & mix-VVS & POTS \\
\hline 3-4 years & 38 & $4(10.5)$ & 0 & 0 & $7(18.4)$ \\
4-5 years & 114 & $12(10.5)$ & 0 & $4(3.5)$ & $7(6.1)$ \\
5-6 years & 193 & $26(13.5)$ & $1(0.5)$ & $10(5.2)$ & $8(4.1)$ \\
Total & 345 & $42(12.2)$ & $1(0.3)$ & $14(4.1)$ & $22(6.4)$ \\
$x^{2}$ value & - & 0.690 & - & 0.236 & 10.854 \\
$P$ & - & 0.708 & - & 0.627 & 0.004
\end{tabular}

WS-V, vasoinhibitory type of vasovagal syncope; WS-C, cardioinhibitory type of vasovagal syncope; mixed-WS, mixed type of vasovagal syncope; POTS, postural tachycardia syndrome.

included $42(12.2 \%)$ cases of vasoinhibitory type VVS, one $(0.3 \%)$ case of cardioinhibitory type VVS, 14 (4.1\%) cases of mixed type VVS, and $22(6.4 \%)$ cases of POTS. There were no hemodynamic responses of $\mathrm{OHT}$ or $\mathrm{OH}$.

\section{Compliance and Safety}

Table 3 showed that eight $(2.3 \%)$ cases did not complete the test due to intolerable sweating, abdominal pain, vomiting, and palpitations. However, none of these patients had severe adverse events during the HUTT process.

\section{Intervention Strategies in Case of Positive Responses}

In case of positive responses, the patients were rapidly placed in the supine position. Strategies including keeping the airways open, oxygen therapy, and intravenous glucose infusion were taken when necessary. All the patients could recover completely within 5 min of rest in the supine position.

\section{DISCUSSION}

The medical history of 345 subjects aged between 3 and 5 years with orthostatic intolerance was reviewed in the present study. 
It was found that the most common symptoms were syncope and dizziness. Sublingual nitroglycerin provocation significantly increased the positive rate of the test compared with only basic HUTT. Vasoinhibitory type VVS was the most frequent positive response to HUTT. Eight patients did not complete the test due to intolerable symptoms, but none of these patients had severe adverse events during the HUTT process.

While upright, $70 \%$ of the blood volume was below the heart (8). Healthy individuals can maintain blood volume after standing due to autonomic and cardiovascular compensatory mechanisms. For patients with orthostatic intolerance, symptomatic hypotension occurs because of hypovolemia due to gravity and a time delay in sympathetic activation (9). BP and cerebral blood flow decrease inappropriately. BP can decrease by over $30 \%$ at $10-15 \mathrm{~s}$ after standing. Symptoms of lightheadedness and reflex tachycardia occur. With the increasing prevalence of orthostatic intolerance in pediatrics and the impairment of the quality of life for such patients, this area of research has drawn increasing attention in recent years.

HUTT has been used to reproduce neurally mediated reflex for years to investigate the heart rate and blood pressure adaptation to position change (10). A study previously reported the HUTT protocol in children aged between 4 and 18 years but did not include children aged below 4 years (11). In a retrospective study, 112 patients (mean age 15.6 years old) with orthostatic intolerance with abnormal HUTT results were included. The results suggested that headaches and syncope were the most frequent symptoms (46 and 29\%, respectively) (4). In a single center, they reported that dizziness was the most common symptom in pediatric patients with POTS (age 5-18 years) (12). In our study, the most frequent symptoms were syncope $(28.7 \%)$ and dizziness $(22.6 \%)$, and $5.5 \%$ of cases had headache symptoms. The inconsistent results may be due to different populations and ages.

In our study, $79(22.9 \%)$ cases had positive responses to complete HUTT (basic HUTT and sublingual nitroglycerin HUTT), while $29(8.4 \%)$ cases had positive responses if only basic HUTT was performed, demonstrating that drug-free HUTT had a low positive rate. It is necessary to perform pharmacological provocation using isoproterenol intravenous infusion or sublingual nitroglycerin (13). A previous study suggested that isoproterenol intravenous infusion significantly increased the sensitivity of the test (from 28 to 45\%) and was associated with a slight decrease in the specificity (from 93 to $86 \%$ ) (14). Sublingual nitroglycerin challenge was safe in children with unexplained syncope with the specificity of $86 \%$ (15). In a meta-analysis, data demonstrated that nitroglycerine administration increased HUTT sensitivity but maintained a similar specificity in comparison to isoproterenol administration (16).

It is critical to identify the tilt angle to maximize the number of true positive responses and to minimize the number of subjects who are falsely assessed as positive responses. In a previous study, it is reported that the positive response appearance time was 15.1 $\pm 14.0 \mathrm{~min}$, and the tilt angle was $60^{\circ}$ in children (11). A metaanalysis confirmed that protocols with the tilt angle of $60^{\circ}$ had a higher specificity associated with a lower sensitivity (16). Also, a tilt angle of $60^{\circ}$ was utilized in our study.

HUTT is safe and well-tolerated. The previous study reported that the most common adverse events were the development of hypertension or tachycardia and intolerable flushing or nausea (17). In a previous study, 13 of 3,488 cases experienced transient aphasia during the HUTT process. The incidence of transient aphasia was higher in adults than in children (18). Approximately one-third of adult patients had some form of arrhythmic event during the HUTT process (19). Severe and rare cardiac complications such as atrial fibrillation was reported in the previous study (20). Eight of our patients discontinued the HUTT due to intolerable sweating, abdominal pain, vomiting, and palpitations. None of our patients suffered neurological and cardiovascular adverse events during the HUTT process.

Patients with orthostatic intolerance have difficulty in tolerating the upright posture and present symptoms and signs when standing, recovering when returning to the supine position. Our 345 patients were diagnosed with orthostatic intolerance according to the clinical manifestation. We further used HUTT to define the hemodynamic responses. For general patients with orthostatic intolerance, non-pharmacologic interventions including health education of avoiding prolonged standing, tilt training, and intake of water and salt were utilized to alleviate the orthostatic intolerant symptom. For patients with positive responses to HUTT, pharmacologic interventions such as midodrine hydrochloride or metoprolol should be used when appropriate (21). HUTT can not only diagnose the hemodynamic type but can also help with the selection of treatment strategies for orthostatic intolerance.

There were some limitations. In our study, $16.5 \%$ of cases presented VVS response with $12.2 \%$ of cases being of the vasoinhibitory type and $6.4 \%$ of cases presented a POTS response to HUTT. However, our study was a retrospective study. Data for healthy individuals aged between 3 and 5 years were not included. The specificity of HUTT for VVS and POTS could not be concluded. Otherwise, the standards for positive responses in children below 6 years of age were controversial.

In conclusion, the positive rate of the HUTT was $22.9 \%$ in pediatric patients aged between 3 and 5 years with orthostatic intolerance. The most common symptoms were syncope and dizziness. The most frequent hemodynamic response to HUTT was vasoinhibitory type VVS. Pharmacological provocation could improve the sensitivity of HUTT. HUTT was safe and well-tolerated for children aged below 6 years.

\section{DATA AVAILABILITY STATEMENT}

The original contributions presented in the study are included in the article/supplementary material, further inquiries can be directed to the corresponding author/s.

\section{ETHICS STATEMENT}

The studies involving human participants were reviewed and HUTT was approved by the Ethics Committee of The Second 
Xiangya Hospital, Central South University. The informed consent was obtained from all the subjects directly or their guardians. Written informed consent to participate in this study was provided by the participants' legal guardian/next of kin. Written informed consent was obtained from the minor(s)' legal guardian/next of kin for the publication of any potentially identifiable images or data included in this article.

\section{AUTHOR CONTRIBUTIONS}

RZ was primarily responsible for the protocol development, patient enrollment, data collecting, preliminary data analysis, and for writing the manuscript. SW assisted with data analysis and critical revision for important content and edited the draft.

\section{REFERENCES}

1. Stewart JM. Common syndromes of orthostatic intolerance. Pediatrics. (2013)131:968-80. doi: 10.1542/peds.2012-2610

2. Stewart JM. Update on the theory and management of orthostatic intolerance and related syndromes in adolescents and children. Expert Rev Cardiovasc Ther. (2012)10:1387-99. doi: 10.1586/erc.12.139

3. Brisinda D, Brocca L, Sorbo AR, Lombardi G, Fioravanti F, Fenici R. Psychophysiological evaluation of patients with transient consciousness loss of uncertain origin. Kardiol Pol. (2018)76:566-73. doi: 10.5603/KP.a2017.0254

4. Gourishankar A, Belton MD, Hashmi SS, Butler IJ, Lankford JE, Numan MT. Demographic and clinical features of pediatric patients with orthostatic intolerance and an abnormal head-up tilt table test: a retrospective descriptive study. Pediatr Neonatol. (2020) 61:68-74. doi: 10.1016/j.pedneo.2019.06.012

5. Chen L, Li X, Todd $\mathrm{O}$, Wang $\mathrm{C}$, Jin $\mathrm{H}$, Du J. A clinical manifestation-based prediction of haemodynamic patterns of orthostatic intolerance in children: a multi-centre study. Cardiol Young. (2014) 24:649-53. doi: 10.1017/S1047951113000929

6. Wang C, Li Y, Liao Y, Tian H, Huang M, Dong X. 2018 Chinese Pediatric Cardiology Society. (CPCS) guideline for diagnosis and treatment of syncope in children and adolescents. Sce Bull. (2018) 63:155864. doi: 10.1016/j.scib.2018.09.019

7. Brignole M, Moya A, de Lange FJ, Deharo JC, Elliott PM, Fanciulli A, et al. 2018 ESC Guidelines for the diagnosis and management of syncope. Eur Heart J. (2018) 39:1883-948. doi: 10.5603/KP.2018.0161

8. Stewart JM, Boris JR, Chelimsky G, Fischer PR, Fortunato JE, Grubb BP, et al. Pediatric disorders of orthostatic intolerance. Pediatrics. (2018) 141:e20171673. doi: 10.1542/peds.2017-1673

9. Sheriff DD, Nadland IH, Toska K. Role of sympathetic responses on the hemodynamic consequences of rapid changes in posture in humans. J Appl Physiol. (1985) (2010) 108:523-32. doi: 10.1152/japplphysiol.011 85.2009

10. Lee SH, Yang JH, Yim HR, Park J, Park SJ, Park KM, et al. Hemodynamic parameters and baroreflex sensitivity during head-up tilt test in patients with neurally mediated syncope. Pacing Clin Electrophysiol. (2017) 40:145461. doi: $10.1111 /$ pace. 13217

11. Lin J, Wang Y, Ochs T, Tang C, Du J, Jin H. Tilt angles and positive response of head-up tilt test in children with orthostatic intolerance. Cardiol Young. (2015) 25:76-80. doi: 10.1017/S1047951113001601

12. Staples A, Thompson NR, Moodley M. Pediatric-onset postural orthostatic tachycardia syndrome in a single tertiary care center. J Child Neurol. (2020) 35:526-35. doi: 10.1177/0883073820916260
FL, PL, JZ, and YW were responsible for completing the headup tilt test. YX and CW supervised the design and execution of the study, checked the data analysis, contributed to the writing of the manuscript and provided final approval of the submitted manuscript. All authors have read and approved the final manuscript and assume full responsibility for its contents.

\section{FUNDING}

This work was supported by grants from Hunan Province Clinical Medical Technology Innovation Guidance Project (2020-236), the Natural Science Foundation of Hunan Province in China (2018JJ3730) and the Health and Family Planning Commission of Hunan Province (20201217).

13. Pietrucha A, Wojewodka-Zak E, Wnuk M, Wegrzynowska M, Bzukala I, Nessler J, et al. The effects of gender and test protocol on the results of head-up tilt test in patients with vasovagal syncope. Kardiol Pol. (2009) 67:1029-34.

14. Lai WT, Chen MR, Lin SM, Hwang HK. Application of head-up tilt table testing in children. J Formos Med Assoc. (2010) 109:6416. doi: 10.1016/S0929-6646(10)60104-0

15. Foglia-Manzillo G, Giada F, Fteita N, Nessi I, Santarone M, Raviele A. Tilt testing potentiated with sublingual nitroglycerin in children with unexplained syncope. Eur Heart J. (2007) 28:2605-09. doi: 10.1093/eurheartj/ehm430

16. Forleo C, Guida P, Iacoviello M, Resta M, Monitillo F, Sorrentino S, et al. Head-up tilt testing for diagnosing vasovagal syncope: a meta-analysis. Int J Cardiol. (2013) 168:27-35. doi: 10.1016/j.ijcard.2012.09.023

17. Graham LA, Gray JC, Kenny RA. Comparison of provocative tests for unexplained syncope: isoprenaline and glyceryl trinitrate for diagnosing vasovagal syncope. Eur Heart J. (2001) 22:497503. doi: 10.1053/euhj.1999.2007

18. Chu W, Wang C, Lin P, Li F, Wu L, Xie Z. Transient aphasia: a rare complication of head-up tilt test. Neurol Sci. (2014) 35:112732. doi: 10.1007/s10072-014-1664-1

19. Prabhu MA, Pillai V, Shenthar J. Comparison of efficacy, pattern of response, occurrence of arrhythmias, and the tolerability of nitroglycerine and isoprenaline as provocative drugs during head-up tilt test. Heart Lung Circ. (2017) 26:586-92. doi: 10.1016/j.hlc.2016.10.006

20. Kim JH, Lee SH, Park SJ, Park KM, On YK, Kim JS. Atrial fibrillation occurring during head-up tilt testing: once detected, atrial fibrillation should be monitored, regardless of how it is detected. Heart Rhythm. (2019)16:5206. doi: 10.1016/j.hrthm.2018.11.030

21. Chen G, Du J, Jin H, Huang Y. Postural tachycardia syndrome in children and adolescents: pathophysiology and clinical management. Front Pediatr. (2020) 8:474. doi: $10.3389 /$ fped.2020.00474

Conflict of Interest: The authors declare that the research was conducted in the absence of any commercial or financial relationships that could be construed as a potential conflict of interest.

Copyright (c) 2021 Zou, Wang, Li, Lin, Zhang, Wang, Xu and Wang. This is an open-access article distributed under the terms of the Creative Commons Attribution License (CC BY). The use, distribution or reproduction in other forums is permitted, provided the original author(s) and the copyright owner(s) are credited and that the original publication in this journal is cited, in accordance with accepted academic practice. No use, distribution or reproduction is permitted which does not comply with these terms. 